\title{
Arbuscular Mycorrhizal Spore Density, Composition and Richness across Four Major Land-use Types in Upper Hantana in Sri Lanka
}

\author{
Mafaziya Nijamdeen T.W.G.F. ${ }^{*}$ and Madawala H.M.S.P. \\ Faculty of Science, University of Peradeniya, Peradeniya, Sri Lanka \\ *fathimamafaziya@gmail.com
}

\begin{abstract}
Arbuscular mycorrhizal (AM) fungi are widespread in different ecosystems and are associated with more than $80 \%$ of all terrestrial species. AM play an influencing role in plant diversity, productivity, community structure and ecosystem processes. Understanding how AM respond to vegetation changes may help understand and interpret the recovery patterns of vegetation. The present study was designed to assess the status of AM spore density, species composition and richness in four major landuse types; Paraserianthus stand (PST) dry patana grassland (DPG) restored pine (RPI) and natural forest patch (NFP) in the Upper Hantana area.
\end{abstract}

From each site 10 soil samples (each is a composite of 3 samples) were taken from $0-20 \mathrm{~cm}$ depth. AM spores were extracted using wet sieving and decanting method and identified to their generic level using keys. A total of 8,234 spores were counted in NFP while 3,721, 1,922 and 1,571 spores were recovered from RPI, DPG and PST respectively. Highest number of spore morphotypes (20) was recorded in NFP, while 18, 16 and 14 were identified from RPI, PST and DPG respectively. Out of the total spore morphotypes, 14, 10, 10 and 6 were identified to their generic levels in NEP, RPI, PST and DPG landuse types respectively. The four landuse types showed contrasting patterns of different-sized spore distribution. NFP had significantly higher densities of larger- $(>125 \mu \mathrm{m})$ and medium-sized spores $(>63 \mu \mathrm{m})$ compared to the other 3 land use types. Glomus was the most abundant genus in all four landuse types. In PST, 5 morphotypes were belonged to the genus Glomus, 3 Acaulospora and 2 Gigaspora. In DPG, 3 Acaulospora spp., 2 Glomus spp. and 1 Scutellospora spp. In RPI, 7 Glomus spp., 3 Gigaspora spp. and in NFP 10 Glomus spp. 2 Gigaspora spp. and 2 Scutellospora spp. were identified. Out of the four landuse types, NFP showed the highest AMF richness with a Shannon-Weiner Index of 2.436 which followed by RPI, DPG and PST (2.280, 2.173 and 2.139 respectively).

The results suggest that the study sites demonstrated distinct AM communities indicating that the vegetation cover and landuse history may play a role in determining the community structure of AM. The higher AM abundance and richness observed in recently restored Pinus stand (RPI) compared to PST and DPG suggest that the AM population can be rapidly restored through increasing plant diversity with native species, which may eventually help restore these ecosystems.

Keywords: Arbuscular mycorrhizae, Dry patana, Land use, Paraserianthus, Restored pine 